\title{
6 Entscheidung über Organspende
}

\author{
Claus-Dieter Middel und Wiebke Abel
}

Das Selbstbestimmungsrecht eines Patienten ist ein hohes und verpflichtendes Gut. Daher muss grundsätzlich in jede medizinische Maßnahme durch den Patienten bzw. durch seinen Patientenvertreter eingewilligt werden. In ausweglos erscheinenden Situationen bei beatmeten Patienten mit schwerer Hirnschädigung stellt sich die Frage, ob eine weitere Behandlung fortzuführen oder aus medizinischen, ethischen und rechtlichen Gründen zu beenden ist. Im besonderen Fall eines unmittelbar bevorstehenden oder vermuteten irreversiblen Hirnfunktionsausfalls muss in der Diskussion zu Therapieentscheidungen und Therapiezielen auch eine mögliche Entscheidung über Organspende berücksichtigt werden. Dies gilt insbesondere vor dem Hintergrund einer Therapiezieländerung mit der Einleitung einer palliativen Behandlung, vor welcher der Gesamtwille des Patienten eruiert werden muss.

\subsection{Wer trifft die Entscheidung für oder gegen eine Organspende?}

Das Transplantationsgesetz (TPG) kodifiziert ein Zusammenwirken von Entscheidungsregelung und erweiterter Zustimmungsregelung:

1. Die Entscheidungsregelung zielt darauf, dass sich ein Patient zu Lebzeiten zur postmortalen Organspende in einer Patientenverfügung oder einer anderen vorsorglichen Willensbekundung (z.B. Organspendeausweis) geäußert hat. Idealerweise wird damit der Patientenwille eindeutig festgelegt: zum einen hinsichtlich des Verhältnisses von Organspende zu palliativmedizinischen Maßnahmen, und zum an- 
deren hinsichtlich der für eine Organspende notwendigen organerhaltenden Maßnahmen.

2. Die erweiterte Zustimmungsregelung greift, sofern keine, und zwar weder positive noch negative schriftliche Erklärungen des Patienten abgefasst wurden bzw. vorliegen. Damit wird ermöglicht, dass die Entscheidung über Organspende subsidiär auch von nächsten Angehörigen oder sonstigen Personen getroffen werden kann. Diese Personen müssen dem potenziellen Organ- oder Gewebespender bis zu seinem Tod in besonderer persönlicher Verbundenheit offenkundig nahegestanden haben oder von ihm die Entscheidung über eine Organ- oder Gewebespende übertragen bekommen haben.

\subsection{Warum kommt bei der Entscheidung für oder gegen eine Organspende der Kommunikation mit den Patientenvertretern und den Angehörigen eine besondere Bedeutung zu?}

Grundsätzlich kommt der Kommunikation mit den Patientenvertretern und den Angehörigen eine besondere Bedeutung zu, da der Gesetzgeber den Sachwaltern des Patienten eine Entscheidungsbefugnis über die Organ- oder Gewebeentnahme einräumt.

Zu Lebzeiten setzt jede medizinische Maßnahme in der Regel die Einwilligung des Patienten nach angemessener Aufklärung voraus. Ist der Patient einwilligungsunfähig, ist eine Einwilligung eines Patientenvertreters einzuholen, soweit keine schriftliche Willensbekundung des Patienten die Maßnahme gestattet oder untersagt. Auch das TPG basiert auf dem Crundsatz, dass erst die Zustimmung des betroffenen Patienten selbst oder seiner nächsten Angehörigen die Organspende legitimiert. Nach dem TPG erfolgt die Zustimmung zur postmortalen Organspende nach $\mathbb{3} 3$ mit Einwilligung des Patienten oder subsidiär nach $\mathbb{} 4$ durch Angehörige oder gleichgestellte Personen.

Liegt keine Zustimmung zur Organspende vor, ist der mutmaßliche Wille des Patienten anhand von konkreten Anhaltspunkten, zu Lebzeiten geäußerten Überzeugungen oder individuellen Wertvorstellungen zu erkunden. In diesen Fällen, in denen mögliche Willensbekundungen des Patienten ausgelegt werden müssen, ist in der Praxis nicht immer und ohne weiteres klar, welche Person stellvertretend die Entscheidung über eine mögliche Organspende zu treffen hat. Dies rührt daher, dass Patientenvertreter und nächster Angehöriger unterschiedliche Personen sein können. Sofern dies 
6.3 Welche Personen können stellvertretend für den Patienten die Entscheidung für oder gegen eine Organspende treffen?

der Fall ist, sind prä-und postmortal unterschiedliche Personen entscheidungsbefugt. Das bedeutet:

- Vor Eintritt des Todes obliegt es dem Patientenvertreter, dem Gesamtwillen des Patienten Ausdruck und Geltung zu verschaffen und hierzu eine harmonisierende Interpretation aller Willensäußerungen herbeizuführen.

- Nach Eintritt des Todes ist nur der nächste Angehörige bzw. die Person abschließend zu befragen, der die Entscheidung über eine Organ- oder Gewebespende übertragen wurde. Im Gegensatz zu der Person, der die Entscheidung über eine Organ- oder Gewebespende übertragen wurde, kann der nächste Angehörige auf sein Entscheidungsrecht verzichten, das dann auf einen nachrangigen Angehörigen übergeht.

Zwar muss die Entscheidung für oder gegen eine Organspende wegen des Ausmaßes intensivtherapeutischer Maßnahmen bereits in einer Phase fallen, in der noch der Patientenvertreter der berufene Ansprechpartner für die zu treffenden Behandlungsentscheidungen ist. Doch müssen im Kontext einer Organspende alle Maßnahmen nicht nur der medizinischen Indikation, sondern auch dem Patientenwillen entsprechen. Daher sollten „in der Zeitspanne im Übergang vom Leben zum Tod“ (Duttge u. Neitzke 2015) Gespräche mit beiden Vertretern über mögliche Therapieoptionen oder Änderungen des Therapieziels geführt werden. Dabei ist zu akzeptieren, dass sich nächste Angehörige ggf. mit der Frage der Organspende in ihrer Trauer oder aus prinzipiellen Erwägungen nicht befassen wollen.

Das heißt: In den Gesprächen über eine mögliche Organ- oder Gewebespende darf kein Entscheidungsdruck aufgebaut werden.

\subsection{Welche Personen können stellvertretend für den Patienten die Entscheidung für oder gegen eine Organspende treffen?}

Zur Entscheidung für oder gegen eine Organ- oder Gewebespende berufen ist der Patientenvertreter oder der nächste Angehörige:

1. Patientenvertreter: Sind Patientenvertreter und nächste Angehörige unterschiedliche Personen, ist der Patientenvertreter bis zur Feststellung des Todes für die Einwilligung in Therapieentscheidungen verantwortlich. Er ist für seine Entscheidungen an den expliziten Willen des Patienten nach $\$ 1901$ a Abs. 1 BGB, seinen mutmaßlichen Willen nach $\mathbb{s} 1901$ a Abs. 2 BGB bzw. sein Wohl nach $\mathbb{1} 1901$ Abs. 2 S. 1 BCB gebunden. Zur Ermittlung des mutmaßlichen Willens soll der 
Patientenvertreter gem. $\mathbb{\$} 1901 \mathrm{~b}$ Abs. 2 BGB den Angehörigen „Gelegenheit zur Äußerung“ geben. Dabei kann es sich um ein Gespräch handeln, dessen Führung und Gestaltung grundsätzlich dem Patientenvertreter obliegt. Er kann dabei den behandelnden Arzt einbeziehen, ist dazu aber nicht verpflichtet.

2. Nächster Angehöriger: Gemeint ist diejenige Person, die in der in $\mathbb{s} 1 \mathrm{a}$ Nr. 5 TPG genannten Reihenfolge an erster Stelle steht (Ehegatte oder eingetragener Lebenspartner, volljährige Kinder, Eltern, Vormund, Pfleger, volljährige Geschwister, Großeltern), innerhalb angemessener Zeit erreichbar ist und in den letzten zwei Jahren vor dem Tod zum Verstorbenen persönlichen Kontakt hatte. Dem nächsten Angehörigen gleichgestellt ist eine volljährige Person, die dem Verstorbenen in besonderer persönlicher Verbundenheit offenkundig nahegestanden hat, z.B. nichteheliche Lebenspartner - diese Person tritt neben den nächsten Angehörigen ( $\$ 4$ Abs. 2 S. 5 TPG). Wurde die Entscheidung über eine Organspende gemäß $\$ 2$ Abs. 2 TPG vom Verstorbenen zu Lebzeiten auf eine namentlich benannte, dritte Person übertragen, so tritt diese an die Stelle des nächsten Angehörigen. Durch diese Regelungen soll sichergestellt werden, dass der nächste Angehörige eine Entscheidung im Sinne des Verstorbenen trifft. Das ist nur gewährleistet, wenn es zu gelegentlichen Treffen oder Gesprächen gekommen ist, wobei der Sterbephase eine besondere Bedeutung zukommt. Im Hinblick auf eine schnelle Entscheidungsfindung ist es ausreichend, wenn einer von zwei gleichrangigen nächsten Angehörigen zustimmt, sofern der andere nicht widerspricht (\$ 4 Abs. 2 S. 3 TPG). Das heißt: Der Widerspruch einer gleichrangigen Person verhindert die Organspende. Ist ein vorrangiger Angehöriger innerhalb angemessener Zeit nicht erreichbar, genügt die Beteiligung und Entscheidung des zuerst erreichbaren nächsten Angehörigen. (\$ 4 Abs. 2 S. 4 TPG). Die zeitliche Grenze, von der an die Unterrichtung des nächsterreichbaren, in der Rangfolge nachgehenden Angehörigen erfolgen darf, ist insbesondere danach zu beurteilen, wie lange nach Eintritt des Todes die betreffenden Organe noch transplantabel sind.

\subsection{Warum muss bei der Entscheidung für oder gegen eine Organspende der Gesamtwille des Patienten ermittelt werden?}

Die Patientenautonomie bestimmt den Blickwinkel, unter dem das Patientenwohl auszulegen ist. Insoweit wäre unter den geltenden rechtlichen Regelungen jede isolierte Bewertung „therapeutischer Interessen“ einerseits 
6.4 Warum muss bei der Entscheidung für oder gegen eine Organspende der Gesamtwille des Patienten ermittelt werden?

und „organprotektiver Interessen“ andererseits von vornherein fragwürdig. Folglich sind der Patientenvertreter oder der nächste Angehörige berufen, stets den Gesamtwillen des Patienten bezüglich einer Organspende zu ermitteln und zur Geltung zu bringen, und zwar mittels einer „Gesamtschau unter Einbeziehung aller Mitentscheidungsbefugten“ (Duttge u. Neitzke 2015).

Dementsprechend wird vorrangig geschaut, ob eine schriftliche Erklärung des Patienten zur Organspende vorliegt, beispielsweise in einem Organspendeausweis oder einer Patientenverfügung. In diesem Fall sind Angehörige ebenso wie die behandelnden Ärzte an die zu Lebzeiten getroffene Entscheidung des Verstorbenen gebunden. Die Einwilligung und die Übertragung der Entscheidung auf Dritte können vom vollendeten 16. Lebensjahr an erklärt werden. Sofern keine schriftliche Erklärung des Patienten zur Organspende vorliegt, hat jede entscheidungsbefugte Person bei der Entscheidung für oder gegen eine Organspende nicht ihre persönliche Auffassung, sondern den mutmaßlichen Willen des Patienten zugrunde zu legen. Erst wenn dieser mutmaßliche Wille des Patienten nicht ermittelbar ist, erfolgt eine Entscheidung nach den Wertvorstellungen der entscheidungsbefugten Person.

Liegt eine Zustimmung zur Organspende vor, hat der Arzt den nächsten Angehörigen gemäß $\$ 3$ Abs. 3 S. 1 TPG über die beabsichtigte Organentnahme zu unterrichten.

Weist der Angehörige oder eine ihm gleichgestellte Person auf eine Aktualisierung oder einen Widerruf der vorliegenden Verfügung hin, so ist dies zu berücksichtigen.

Ist der mutmaßliche Wille des Patienten nicht zu ermitteln, trifft der nächste Angehörige oder eine ihm gleichgestellte Person in Ausübung des Totensorgerechts eine subsidiäre eigenständige Entscheidung, welche die fehlende Willensäußerung des Verstorbenen ersetzt.

Liegt keine Zustimmung zur Organspende vor, ist die Entnahme nicht zulässig. Dies gilt auch dann, wenn im Falle eines nicht dokumentierten Patientenwillens niemand zur Vertretung des Patienten befugt ist oder die befugten Personen nicht in angemessener Zeit kontaktierbar sind.

Das heißt: Die Einwilligung ist unabdingbare Voraussetzung für die Organspende. 


\title{
6.5 Wer oder was könnte in der Praxis bei der Ermittlung des Gesamtwillens des Patienten noch hilfreich sein?
}

\begin{abstract}
Zur Vermeidung von Konflikten oder gar gerichtlichen Auseinandersetzungen kann es sich in emotional sehr belastenden Situationen, in denen schwierige Entscheidungen zu treffen sind, als hilfreich erweisen, eine Ethik-Fallberatung in Anspruch zu nehmen (z.B. Ethikkonsil, klinisches Ethikkomitee). Wird hinsichtlich des Gesamtwillens des Patienten kein Konsens erzielt, ist letztlich das Betreuungsgericht zur Entscheidung berufen ( $\$ 1904$ BGB). Im Sinne der Patientenautonomie müssten dann bis zur Entscheidung des Gerichts die Organfunktionen durch intensivmedizinische Maßnahmen so aufrechterhalten werden, dass einem möglichen Organspendewunsch entsprochen werden könnte.
\end{abstract}

\section{Literatur}

Bundesärztekammer, Zentrale Ethikkommission bei der Bundesärztekammer (2013) Empfehlungen der Bundesärztekammer und der Zentralen Ethikkommission bei der Bundesärztekammer: Umgang mit Vorsorgevollmacht und Patientenverfügung in der ärztlichen Praxis. Deutsches Ärztebl 110(33/34), 1580.URL: http://www.aerzteblatt.de/archiv/145276 (abgerufen am 28.06.2021)

Bundesärztekammer, Zentrale Ethikkommission bei der Bundesärztekammer (2013) Arbeitspapier zum Verhältnis von Patientenverfügung und Organspendeerklärung. Deutsches Ärztebl 110, 2013(12), A572-A574. URL: https://www.aerzteblatt.de/pdf.asp?id=135909 (abgerufen a, 28.06.2021)

Deutsche Interdisziplinäre Vereinigung für Intensiv- und Notfallmedizin (2015) Entscheidungen bei potentiellen Organspendern. Gemeinsames Positionspapier der Sektion Ethik und der Sektion Organspende und -transplantation der DIVI. URL: https://www.divi.de/stellungnahmen/publikationen/stellungnahmen/gemeinsames-positionspapier-der-sektion-ethik-und-der-sektion-organspende-und-transplantation-der-divi-zu-entscheidungen-bei-potentiellen-organspendern

Deutsche Interdisziplinäre Vereinigung für Intensiv- und Notfallmedizin (2016) Grenzen der Sinnhaftigkeit von Intensivmedizin. Positionspapier der Sektion Ethik der DIVI. Medizinische Klinik - Intensivmedizin und Notfallmedizin. DOI:10.1007/s00063-016-0202-8

Deutsche Interdisziplinäre Vereinigung für Intensiv- und Notfallmedizin (2012) Therapiezieländerung und Therapiebegrenzung in der Intensivmedizin. Positionspapier der Sektion Ethik der DIVI. URL: https://www.divi.de/empfehlungen/publikationen/ethik/therapiezielaenderung-und-therapiebegrenzung

Deutscher Ethikrat (2015) Hirntod und Entscheidung zur Organspende. Stellungnahme. Berlin. URL: https://www.ethikrat.org/fileadmin/Publikationen/Stellungnahmen/deutsch/stellungnahmehirntod-und-entscheidung-zur-organspende.pdf

Duttge G, Neitzke G (2015) Zum Spannungsfeld zwischen Intensivtherapie und Organtransplantation, GesR 705-709

Middel CD, Scholz K (2018) Gesetz über die Spende, Entnahme und Übertragung von Organen und Geweben (Transplantationsgesetz - TPG). In: Spickhoff A (Hrsg.) Medizinrecht, 3. Aufl., München 